\title{
DYES WITH HIGH DIELECTRIC CONSTANTS
}

\author{
H. LANGHALS \\ Institut für Organische Chemie der Universität München, Karlstrasse 23, D-8000 Munich 2, Federal Republic of Germany
}

Received 27 May 1988; in final form 4 July 1988

\begin{abstract}
The dielectric constants of perylene dyes, perylene-3,4:9,10-tetracarboxylic bisimides, are reported. With aromatic substituents, dielectric constants up to 110 are obtained. With polymeric dyes, the dielectric constants rise to 260 . Mechanisms and applications are discussed.
\end{abstract}

\section{Introduction}

Materials with high dielectric constants find wide application in electronic devices, for instance in capacitors, wave guides, and reflective materials for electromagnetic radiation. For very high dielectric constants materials with ion or dipole lattices are usually used, for example the dielectrics in tantalum capacitors. However, these materials have the disadvantage of a rather strong coupling of electrical charging to lattice vibrations. This mechanism can cause dielectric loss at high frequencies [1].

Better results can be expected when the dielectrics consist of electrically neutral molecules with a low dipole moment. If they can be easily polarized, a high dielectric constant will result. Large conjugated $\pi$ systems are highly polarizable and form the basis of the chromophores in dyes. Therefore, clectrically neutral dyes are especially suitable as dielectrics. The dielectric constants of perylene dyes 1 , perylene$3,4: 9,10$-tetracarboxylic bis-imides, are examined in this paper.

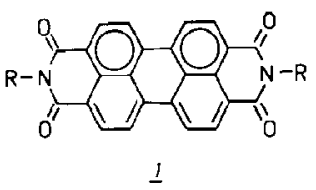

\section{Experimental details}

Measurements are carried out using pressed cylinders of diameter $13 \mathrm{~mm}$ and 0.45 to $1.55 \mathrm{~mm}$ thickness. These were obtained from polycrystalline material by a force of 10 ton (corresponding to a pressure of 7400 bar) lasting for 5-10 min. During this time the samples are evacuated in order to prevent occlusion of air.

For the determination of dielectric constants the pressed cylinders are contacted with silver at the circular surfaces and the capacity of this arrangement was measured at room temperature with a compensating bridge (BR-8 of Belco) at $1.2 \mathrm{kHz}$.

The resistivity of the dyes are measured by use of a de voltage of $15 \mathrm{~V}$. The contacted pressed cylinders and a voltmeter with a dc impedance of $1 \mathrm{M} \Omega$ are serially connected to the power supply and the specific resistance is calculated from the voltage drop at the voltmeter. The specific resistance is constant during $5 \mathrm{~min}$ and the analogous measurement with a voltmeter of $10 \mathrm{M} \Omega$ gives the same specific resistance within experimental error.

Dielectric constants and specific resistances are given in table 1 .

The perylene dyes have been prepared and purified according to the literature [2-4] by condensation of the corresponding primary amines with perylene-3,4:9,10-tetracarboxylic bisanhydride (2).

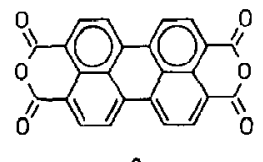

$\underline{2}$

The synthesis of polymeric perylene dyes is more 
Table 1

Dielectric constants and resistivities of dyes

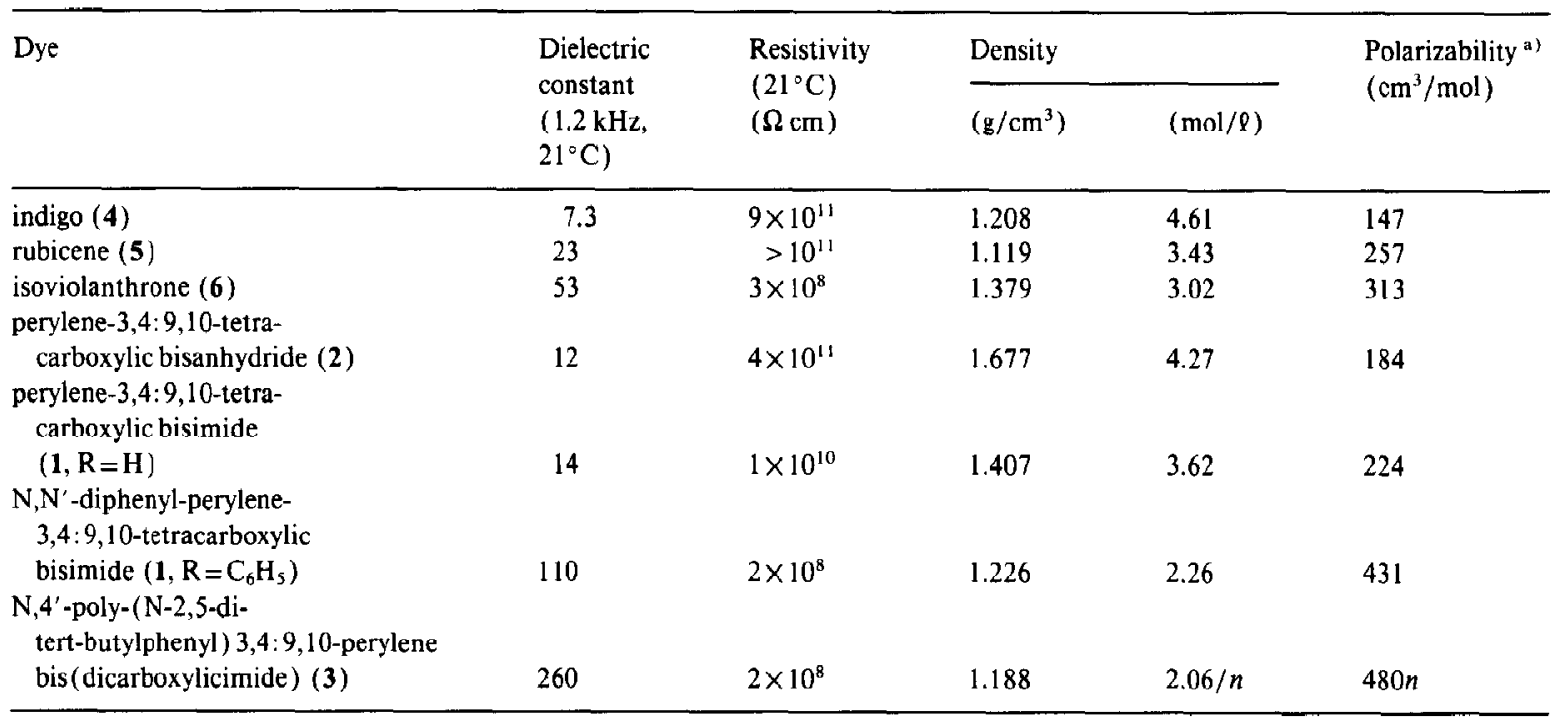

a) Molecular polarizabilities calculated by means of the Clausius-Mossotti equation.

difficult than that of monomers. They are prepared by condensation of a diamine with the anhydride 2 . The degree of polymerization $P_{n}$ as a function of the conversion $p$ is given by Carother's equation [5]

$P_{n}=1 /(1-p)$

with the prerequisite that both the diamine and 2 are exactly equimolar. For high degrees of polymerization a nearly complete conversion is necessary. However, this cannot be attained in quinoline solution [6], but molten imidazole [3] as a solvent gives good results. Further improvements are obtained by substitution of the aromatic diamines with tert-butyl groups, which render the oligomers soluble $[7,8]$ during the polymerization process. Under these conditions the conversion rate is so high that signals of anhydride groups cannot be found in the IR spectrum.

After polymerization, the tert-butyl groups can be removed by a thermal retro Friedel Crafts reaction at temperatures of about $250-450^{\circ} \mathrm{C}$.

$N, 4^{\prime}$-poly-(N-2,5-di-tert-butylphenyl)3,4:9,10perylenebis(dicarboxylic imide) (3).

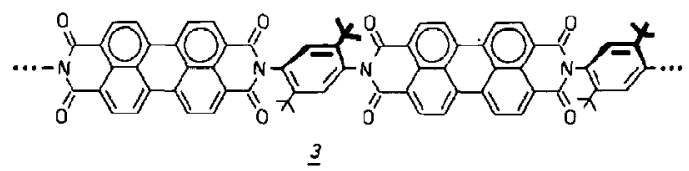

$280.9 \mathrm{mg}$ (1.275 mmol) 1,4-diamino-2,5-di-tertbutylbenzene [9], $500.0 \mathrm{mg}(1.275 \mathrm{mmol})$ perylene-3,4:9,10-tetracarboxylic bisanhydride (2), 150 mg zinc acetate, and $2.5 \mathrm{~g}$ imidazole were heated at $190^{\circ} \mathrm{C}$ under $\mathrm{N}_{2}$ for $16 \mathrm{~h}$. The reaction product was treated with $100 \mathrm{ml} 60 \%$ ethanol/water, filtered, washed with ethanol, and then treated with $5 \%$ hot potassium carbonate. The product was washed with hot water and dried. This yielded $630 \mathrm{mg}(86 \%)$ dark brown solid with the melting point $>360^{\circ} \mathrm{C}$ and density of $1.188 \mathrm{~g} / \mathrm{cm}^{-3}$. IR(KBr $): \nu\left(\mathrm{cm}^{-1}\right)=2965$ w, 1704 s, 1667 s, $1593 \mathrm{~s}, 1577 \mathrm{~s}, 1498 \mathrm{~m}, 1430 \mathrm{~m}$, $1402 \mathrm{~m}, 1341 \mathrm{~s}, 1248 \mathrm{~m}, 1176 \mathrm{~m}, 1088 \mathrm{w}, 953 \mathrm{w}, 850$ w, $811 \mathrm{~m}, 749 \mathrm{w}, 667 \mathrm{w}, 655 \mathrm{w}$ - no detectable absorption at 1773,1756 , and $1730 \mathrm{~cm}^{-1}$ (2). UV (pyridine): $\lambda_{\text {max }}\left(\epsilon_{\text {rel }}\right)=435(0.14), 464(0.27)$, $496(0.64), 533 \mathrm{~nm}(1.0) . \mathrm{UV}\left(\mathrm{H}_{2} \mathrm{SO}_{4}\right): \lambda_{\text {max }}\left(\epsilon_{\mathrm{rel}}\right)$ $=524(0.23), 566(0.55), 612 \mathrm{~nm}$ (1.0). Fluorescence $($ pyridine $): \lambda_{\max }=628 \mathrm{~nm}$.

\section{Results and discussion}

The effect of the polarizability of $\pi$ electrons on the macroscopic dielectric constant is examined with dye molecules which are centrosymmetric and therefore have no permanent dipole moments. They can 
only act through the induced polarization. In order to get high polarizabilities, electronic transitions should be at long wavelengths with high oscillator strengths [1].

A dye molecule with a rather small chromophore which fulfils this condition is indigo (4), which has a dielectric constant of 7.3 (see table 1). Rubicene (5) has a higher value in accordance with its larger $\pi$ system and therefore its higher polarizability.
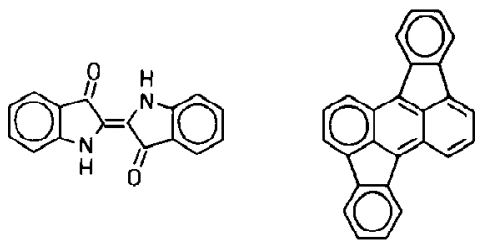$$
\leq
$$

$\underline{5}$

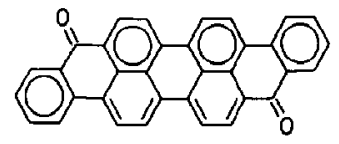

$\underline{6}$

The centrosymmetric vat dye isoviolanthrone (6) has an even larger conjugated $\pi$ system and the dielectric constant of the dye attains the high value of 53.

The perylene dyes 1 have large conjugated centrosymmetric $\pi$ systems. The dielectric constant of 1 with $R=H$ is 14 . This value represents the chromophore itself and is about the same for the anhydride $2(\epsilon=12)$ which has similar $\pi$ orbitals.

Addition of further $\pi$ systems to $1, R=\mathrm{C}_{6} \mathrm{H}_{5}$, increases the dielectric constant to 110 . These groups are twisted out of the plane of the chromophore $[2,10]$ and the conjugation is low. Therefore, these additional groups increase the dielectric constant only by electrostatic interactions.

In order to get even higher dielectric constants by this mechanism one can use a cooperative process in the way that one induced dipole amplifies the next one by electrostatic interactions. For this purpose, the dyes must be closely tied together as in fig. 1 . However, a direct contact of the chromophores must be avoided, for conjugation gives a very large $\pi$ system which can have the properties of an organic metal [11].

This problem is solved by putting p-phenylene links

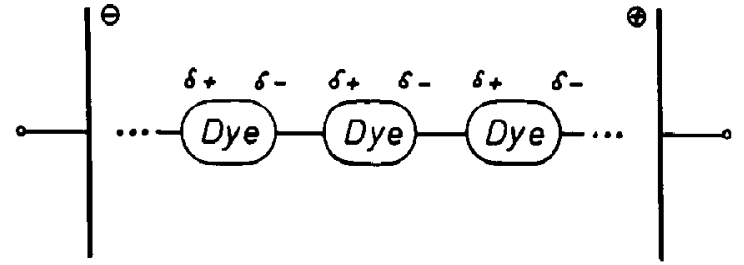

Fig. 1. Arrangement of dyes for the electrostatic interaction of the induced dipoles.

between the perylene chromophores as insulators. They are placed exactly orthogonal to the chromophores by the steric interaction of tert-butyl groups in the $\sigma$-positions. The presence of these tert-butyl groups has further advantages in the polymerization reaction as given in the experimental part. The resulting polymeric dye 3 has all the prerequisites for a high dielectric constant. The experimentally determined value is 260 , which is extraordinarily high for a solid not consisting of ions or dipoles.

\section{Conclusions}

Polymeric perylene fluorescent dyes with insulating links between the chromophores form unpolarized solids with extraordinarily high dielectric constants. The insulation resistance is high enough (see table 1) to make these materials useful as dielectrics. They might be superior to materials with dye ion lattices [12]. It can be expected that the high dielectric constants of perylene dyes exist at very high frequencies, because they are based on electron mobility, which is indicated by their strong IR reflection [13].

They also have an extraordinarily high photostability and are very resistant to chemicals and radiation. For example, a $50 \mu \mathrm{A}$ and $50 \mathrm{keV}$ electron beam focused to $10000 \AA^{2}$ will not cause any detectable decomposition within 3 min radiation time. These properties are important for practical applications of the dyes as dielectrics.

\section{Acknowledgement}

This work was supported by Stiftung Volkswagenwerk and Fonds der Chemischen Industrie. 


\section{References}

[1]C. Kittel, Einführung in die Festkörperphysik, Vol. 4 (Oldenburg, Munich, 1972) p. 541.

[2] A. Rademacher, S. Märkle and H. Langhals, Chem. Ber. 115 (1982) 2927.

[3] H. Langhals, Chem. Ber. 118 (1985) 4641.

[4] S. Demmig and H. Langhals, Chem. Ber. 121 (1988) 225.

[5] J.M.G. Cowie, Chemie und Physik der Polymeren, Vol. 1 (Verlag Chemie, Weinheim, 1976) p. 28.

[6] G. Geissler and H. Remy, Hoechst AG, D.O.S. 1130099 (October 24, 1959) (Chem. Abstr. 57 (1962) pl13461f).

[7] H. Langhals, D.O.S. 3016764 (April 30, 1980) (Chem. Abstr. 96 (1982) 70417x).
[8] H. Langhals, Nachr. Chem. Tech. Lab. 28 (1980) 716 (Chem. Abstr. 95 ( 1981 ) R9816q).

[9] A.J. Hoefnagel, J.H.A.J. Nunnink, A. van Veen, P.E. Verkade and B.M. Wepster, Rec. Trav. Chim. 88 (1969) 386.

[10] G. Bott, L.D. Field and S. Sternhell, J. Am. Chem. Soc. 102 (1980) 5618.

[11] J.H. Hodgkin, J. Di Bari and W.A. Little, US Clearinghouse Fed. Sci. Tech. Inform. AD1971, 726200 (Chem. Abstr. 76 (1972) 14976d).

[12] S. Ikeno, H. Mikawa and M. Yokoyama, Japan Kokai 7662,840 (November 28, 1974) (Chem. Abstr. 85 (1976) $135980 \mathrm{v})$.

[13] F. Graser, D.O.S. 3,422,757 (January 2, 1986) (Chem. Abstr. 105 (1986) $210415 \mathrm{~b}$ ). 